\title{
Spatial structure of internal tidal waves generated on weak topographies
}

\author{
Ping Fan ${ }^{\mathrm{a}, *}$, Vijay P. Singh ${ }^{\mathrm{b}, \mathrm{c}}$ \\ a Institute of Mechanics, Chinese Academy of Sciences, No. 15 Beisihuanxi Road, Beijing 100190, China \\ ${ }^{b}$ Department of Biological and Agricultural Engineering, Texas A \& M University, College Station, TX 77843-2117, USA \\ c Department of Civil \& Environmental Engineering, Texas A \& M University, College Station, TX 77843-2117, USA
}

\section{A R T I C L E I N F O}

\section{Article history:}

Received 31 October 2008

Received in revised form 10 June 2009

Accepted 19 June 2009

Available online 27 June 2009

\section{Keywords:}

Stratified flow

Barotropic tide

Internal waves

Coriolis force

\begin{abstract}
A B S T R A C T
The generation of internal gravity waves by barotropic tidal flow passing over a two-dimensional topography is investigated. Rather than calculating the conversion of tidal energy, this study focuses on delineating the geometric characteristics of the spatial structure of the resulting internal wave fields (i.e., the configurations of the internal beams and their horizontal projections) which have usually been ignored. It is found that the various possible wave types can be demarcated by three characteristic frequencies: the tidal frequency, $\omega_{0}$; the buoyancy frequency, $N$; and the vertical component of the Coriolis vector or earth's rotation, $f$. When different possibilities arising from the sequence of these frequencies are considered, there occur 12 kinds of wave structures in the full 3D space in contrast to the 5 kinds identified by the 2D theory. The constant wave phase lines may form as ellipses or hyperbolic lines on the horizontal plane, provided the buoyancy frequency is greater or less than the tidal frequency. The effect that stems from the consideration of the basic flow is also found, which not only serves as the reason for the occurrence of higher harmonics but also increases the wave strength in the direction of basic flow.
\end{abstract}

(c) 2009 Elsevier B.V. All rights reserved.

\section{Introduction}

The generation of internal gravity waves in deep oceans is a subject of increasing research interest and has attracted considerable attention over the years (Garrett and Kunze, 2007). These waves,

\footnotetext{
* Corresponding author. Tel.: +86 10 82544171; fax: +86 1062561284.

E-mail addresses: fanping@imech.ac.cn (P. Fan), vsingh@tamu.edu (V.P. Singh).
} 
known as internal tides, are generated by the tidal flow in a density-stratified ocean over its bottom topography and the first stage in understanding their effect is to quantify the process of generation. For decades, the processes of tidal generation and attendant energy conversion have been thought to significantly contribute to the mixing in the deep ocean and catalyzing the thermohaline circulation (Munk and Wunsch, 1998). Most theoretical studies up-to-date have focused largely on the tidal energy conversion between the barotropic tide and the internal tide (e.g., Bell, 1975a,b; Balmforth et al., 2002; Smith and Young, 2002, 2003). Recently, Garrett and Kunze (2007) presented a review of the energy conversion studies. On the other hand, limited attention has been directed at the spatial structure of the resulting internal wave fields, especially in a 3D space, which is also crucial for the understanding of the generation process of internal tides (Bühler and Muller, 2007).

In recent years, activities of semi-diurnal internal tides have been observed above the critical latitude (Pisarev, 1996; Parsons et al., 1996; Vlasenko et al., 2003), where freely propagating internal waves are not accommodated by the traditional linear theory of baroclinic tides. Factors of nonlinearity and convection are regarded as the principle reasons for this limitation (Nakamura et al., 2000; Nakamura and Awaji, 2001; Vlasenko et al., 2003). Besides, another reason that lies in the inclusion of the horizontal component of the earth's rotation ('non-traditional' term) seems more convincing (Fan and Singh, 2008). It has been theoretically shown that the non-traditional effect can change the direction of propagation of internal wave energy. The internal beams are no longer symmetric to the vertical and can propagate across the critical latitude, if the gyroscopic waves prevail (LeBlond and Mysak, 1978). These findings indicate that the methods that neglect the traditional term are incapable to elucidate the internal wave structure in extreme conditions. As a consequence, non-traditional effects have received renewed attention due to their unique role in ocean dynamics.

The effects of non-traditional terms for two-dimensional wave structures are now well known, which lead to the occurrences of the asymmetric internal wave rays (Durran and Bretherton, 2004; Fan and Singh, 2008). Under non-traditional approximations, propagation of internal wave rays has five kinds of modes, which can be classified by comparing the magnitudes of frequencies, including the buoyancy frequency, $N$; the tidal frequency, $\omega_{0}$; and the vertical component of the earth's rotation, $f$ (Fan and Singh, 2008). On the other hand, our understanding of the internal tide generation in a 3D space is limited (Garrett and Kunze, 2007). Our understanding of the special attributes of non-traditional terms can be attributed to LeBlond and Mysak (1978), who showed that the constant frequency surfaces can form three kinds of wave types in rotated coordinates under non-traditional approximations. However, the actual wave structures in a wider parameter space close to a real ocean are still unclear. Thus, there is a need to have a comprehensive discussion of the 3D structures of internal waves taking into account some special external factors, such as non-horizontal terms of the Coriolis force and the basic tidal flow.

For an exact description of wave structures, especially in complicated environmental conditions, an evaluation of the wave fields is indispensable. In practice, many efficient methods have been proposed for dealing with a steady-state internal gravity wave problem (e.g., Hurley, 1972; Lighthill, 1967, 1978; Voisin, 1991). The objective of this study is therefore to develop a more systematic and generalized procedure using Lighthill's theory $(1967,1978)$, solve the Fourier integrals involved in the aforementioned problem, and find the asymptotic far-field expressions for these waves. In the analysis here, the basic tidal flow $\mathbf{U}$ is retained, although it is considered to be uniform in the vertical direction. The horizontal component of earth's rotation, known as 'non-traditional approximation,' is included. These two factors are usually ignored for a 3D study on the internal tide generation, which, however, are always the concomitants of tidal movements, and hence their effects need to be evaluated.

The paper is organized as follows. Sections 2 and 3 describe the model and the dispersion relation. In Section 4 the wave fields for a homogeneous ocean are derived with the aid of Lighthill's theory and compared with the results of a 2D representation. This includes descriptions of the wave fields in three conditions that the buoyancy frequency larger than, less than or equal to the tidal frequency, and the demarcation criteria for each style of wave structures. The conclusions are summarized in Section 5. 


\section{Model equation}

The linearized equations governing the motion of a density-stratified Boussinesq fluid of ambient density $\rho_{0}(z)$ on the non-traditional $f$-plane are

$$
\begin{aligned}
& D u-f v+f_{h} w=-\frac{1}{\rho_{0}} p_{x}, \quad D v+f u=-\frac{1}{\rho_{0}} p_{y}, \quad D w-f_{h} u=-\frac{1}{\rho_{0}} p_{z}-\frac{\rho}{\rho_{0}} g, \\
& u_{x}+v_{y}+w_{z}=0, \quad D \rho=\frac{\rho_{0}}{g} N^{2}(z) w
\end{aligned}
$$

where

$$
D=\frac{\partial}{\partial t}+U \frac{\partial}{\partial x}+V \frac{\partial}{\partial y}, \quad \boldsymbol{U}=[U, V]=\left[U_{0} \cos \omega_{0} t, \quad V_{0} \cos \omega_{0} t\right]
$$

In (1) and (2), $N(z)$ denotes the buoyancy frequency; $\rho$ is the perturbed density; $p$ is the pressure; and $g$ is the gravitational acceleration; $x$ (west-east), $y$ (south-north) and $z$ (vertical, positive upward, with the origin at the undisturbed surface) are the Cartesian coordinates; and $u, v, w$ are the corresponding velocity components. The Coriolis vector can be represented as $\left(0, f_{h}, f\right)=2 \boldsymbol{\Omega}=2 \boldsymbol{\Omega}(0, \cos \theta, \sin \theta)$, in which $\boldsymbol{\Omega}$ is the angular velocity of the earth; and $\theta$ is the latitude. In the traditional approximation, one would take $f_{h}=0$.

As stated clearly by Vallis (2006), it is convenient to express the above governing equations in the spherical coordinates when phenomena on a scale comparable to global are considered. Under this situation, the traditional approximation is confirmed to be necessary for the conservation of angular momentum and energy in conjunction with the shallow-fluid approximation. In this paper, as the internal tides operate on a scale smaller than the global scale for which the use of spherical coordinates becomes awkward, it is more convenient to use a local Cartesian representation of the equations. Multiplying the momentum equations individually by $u, v$ and $w$ gives:

$$
u D u-f u v+f_{h} u w=-\frac{1}{\rho_{0}} u p_{x}, \quad v D v+f u v=-\frac{1}{\rho_{0}} v p_{y}, \quad w D w-f_{h} u w=-\frac{1}{\rho_{0}} w p_{z}-\frac{\rho}{\rho_{0}} g w
$$

Adding up the above equation yields:

$$
\frac{1}{2} \rho_{0} D\left(u^{2}+v^{2}+w^{2}\right)=-\boldsymbol{U} \cdot \boldsymbol{F}
$$

where the vector $\mathbf{U}$ represents the velocity and $\mathbf{F}$ denotes the effective force defined by $\mathbf{F}=(\nabla p+\rho g)$. Eq. (4) makes clear that the consideration of horizontal component of the earth's rotation does not disobey the principle of conservation of energy.

Eq. (1) can be reduced to an equation for the vertical velocity, $w$, as:

$$
\left(D^{2}+f^{2}\right) w_{z z}+2 f_{h} w_{y z}+\left\{\left[N^{2}(z)+D^{2}\right] \boldsymbol{\Delta}_{h}+f_{h}^{2} \partial_{y y}\right\} w=0
$$

where $\boldsymbol{\Delta}=\partial_{x x}+\partial_{y y}+\partial_{z z}, \boldsymbol{\Delta}_{h}=\partial_{x x}+\partial_{y y}$. The linearized upper and lower boundary conditions for the rigid surface $z=0$ and bottom $z=-h_{0}$ are

$$
\begin{aligned}
& w=0, \quad z=0 ; \\
& w=\boldsymbol{U} \cdot \nabla H, \quad z=-h_{0}
\end{aligned}
$$

where $H(x)$ is the amplitude of topography. Introduction of the following transformations:

$$
\xi=x-\int_{0}^{t} U(\tau) d \tau=x-\frac{U_{0}}{\omega_{0}} \sin \omega_{0} t, \quad \zeta=y-\int_{0}^{t} V(\tau) d \tau=y-\frac{V_{0}}{\omega_{0}} \sin \omega_{0} t, \quad \eta=t
$$

yields

$$
\begin{aligned}
& \left(\partial_{\eta \eta}+f^{2}\right) W_{z z}+2 f f_{h} \partial_{\zeta} W_{z}+\left\{\left[N^{2}(z)+\partial_{\eta \eta}\right] \boldsymbol{\Delta}_{h}{ }^{\prime}+f_{h}^{2} \partial_{\zeta}{ }^{2}\right\} W=0 \\
& W=0, \quad z=0 \\
& W=\left(U H_{1 \xi}+V H_{1 \zeta}\right), \quad z=-h_{0}
\end{aligned}
$$


where $W(\xi, \zeta, z, \eta)$ and $H_{1}(\xi, \zeta, z, \eta)$ are the vertical velocity and height of the topography in the moving frame, $\boldsymbol{\Delta}_{h}{ }^{\prime}=\partial_{\xi \xi}+\partial_{\zeta \zeta}$.

The objective here is to deduce the internal wave fields on the basis of (8) and discuss the unique spatial structures of the resulting wave fields that are still unknown. Throughout the analysis, the amplitude of topography is assumed to be much less than the depth of ocean, which enables us to apply the bottom boundary condition at the flat surface $z=-h_{0}$ rather than at the actual position of the bottom boundary $z=-h_{0}+H(x)$.

\section{Dispersion relation}

In order to solve (8), we use the two-dimensional Fourier transform with respect to $\xi$ and $\zeta$, defined as:

$$
\overline{\bar{W}}\left(k_{1}, k_{2}\right)=\frac{1}{2 \pi} \int_{-\infty}^{\infty} \int_{-\infty}^{\infty} W(\xi, \zeta) e^{-i\left(k_{1} \xi+k_{2} \zeta\right)} d \xi d \zeta
$$

Thus, we obtain the following boundary value problem:

$$
\begin{aligned}
& \left(\partial_{\eta \eta}+f^{2}\right) \overline{\bar{W}}_{z z}+2 i f f_{h} k_{2} \overline{\bar{W}}_{z}-\left\{\left[N^{2}(z)+\partial_{\eta \eta}\right] k_{h}^{2}+f_{h}^{2} k_{2}^{2}\right\} \overline{\bar{W}}=0 ; \\
& \overline{\bar{W}}=0, \quad z=0
\end{aligned}
$$

where $k_{h}^{2}=k_{1}^{2}+k_{2}^{2}$. Furthermore, according to the definition of the Fourier transform, the bottom function can be expressed as:

$$
\begin{aligned}
H(x, y) & =\frac{1}{2 \pi} \int_{-\infty}^{\infty} \int_{-\infty}^{\infty} \overline{\bar{H}}\left(k_{1}, k_{2}\right) e^{i\left(k_{1} x+k_{2} y\right)} d k_{1} d k_{2} \\
& =\frac{1}{2 \pi} \int_{-\infty}^{\infty} \int_{-\infty}^{\infty} \overline{\bar{H}}\left(k_{1}, k_{2}\right) e^{i\left(k_{1} \int_{0}^{\eta} U d \tau+k_{2} \int_{0}^{\eta} V d \tau\right)} e^{i\left(k_{1} \xi+k_{2} \zeta\right)} d k_{1} d k_{2} \\
& =H_{1}(\xi, \zeta) \frac{1}{2 \pi} \int_{-\infty}^{\infty} \int_{-\infty}^{\infty} \overline{\bar{H}}_{1}\left(k_{1}, k_{2}\right) e^{i\left(k_{1} \xi+k_{2} \zeta\right)} d k_{1} d k_{2}
\end{aligned}
$$

It is easy to find

$$
\overline{\bar{H}}_{1}\left(k_{1}, k_{2}\right)=\overline{\bar{H}}\left(k_{1}, k_{2}\right) e^{i\left(k_{1} \int_{0}^{\eta} U d \tau+k_{2} \int_{0}^{\eta} V d \tau\right)}=\overline{\bar{H}}\left(k_{1}, k_{2}\right) e^{i \kappa \sin \omega_{0} t}
$$

where

$$
\kappa=\frac{k_{1} U_{0}+k_{2} V_{0}}{\omega_{0}}
$$

denotes the tidal excursion parameter. Invoking the Bessel function

$$
e^{i x \sin \theta}=\sum_{n=-\infty}^{\infty} J_{n}(x) e^{i n \theta}, \quad e^{i x \cos \theta}=\sum_{n=-\infty}^{\infty} i^{n} J_{n}(x) e^{i n \theta}
$$

where $J_{n}$ is a Bessel function of order $n$, (12) can be rewritten as:

$$
\overline{\bar{H}}_{1}\left(k_{1}, k_{2}\right)=\overline{\bar{H}}\left(k_{1}, k_{2}\right) \sum_{n=-\infty}^{\infty} J_{n}(\kappa) e^{i n \omega_{0} \eta}
$$

Therefore, the Fourier transform of the bottom boundary condition is

$$
\begin{aligned}
\overline{\bar{W}}\left(-h_{0}\right) & =i\left(k_{1} U+k_{2} V\right) \overline{\bar{H}}_{1}\left(k_{1}, k_{2}\right)=\overline{\bar{H}}\left(k_{1}, k_{2}\right) \frac{\partial}{\partial \eta} e^{i\left(k_{1} \int_{0}^{\eta} U d \tau+k_{2} \int_{0}^{\eta} V d \tau\right)} \\
& =\overline{\bar{H}}\left(k_{1}, k_{2}\right) \sum_{n=-\infty}^{\infty} i n \omega_{0} J_{n}(\kappa) e^{i n \omega_{0} \eta}
\end{aligned}
$$


Following Bell (1975a) and Khatiwala (2003), the BC (16) motivates us to seek a series solution of the form:

$$
\overline{\bar{W}}\left(k_{1}, k_{2}, z, \eta\right)=\overline{\bar{H}}\left(k_{1}, k_{2}\right) \sum_{n=-\infty}^{\infty} w_{n}\left(k_{1}, k_{2}, z\right) J_{n}(\kappa) e^{i n \omega_{0} \eta}
$$

Substituting (17) into (10), we have

$$
\begin{aligned}
& \left(n^{2} \omega_{0}^{2}-f^{2}\right) \frac{d^{2} w_{n}}{d z^{2}}-2 i f f_{h} k_{2} \frac{d w_{n}}{d z}+\left\{\left[N^{2}(z)-n^{2} \omega_{0}^{2}\right] k_{h}{ }^{2}+f_{h}{ }^{2} k_{2}{ }^{2}\right\} w_{n}=0 ; \\
& w_{n}(0)=0 ; \quad w_{n}\left(-h_{0}\right)=i n \omega_{0}
\end{aligned}
$$

Note that the above ODE is ill-posed, provided that $n^{2} \omega_{0}^{2}=f^{2}$. Thus, with the assumption of $n^{2} \omega_{0}^{2} \neq f^{2}$ and introducing the following transformation:

$$
w_{n}(z)=\varphi(z) \exp \left(\frac{i f f_{h} k_{2}}{n^{2} \omega_{0}^{2}-f^{2}} z\right)
$$

one obtains the following boundary value problem for $\varphi$ :

$$
\begin{aligned}
& \varphi_{z z}+K^{2}(z) \varphi=0 \\
& \varphi(0)=0, \quad \varphi\left(-h_{0}\right)=i n \omega_{0} \exp \left(\frac{i f f_{h} k_{2}}{n^{2} \omega_{0}^{2}-f^{2}} h_{0}\right)
\end{aligned}
$$

Here, the vertical wave number $K(z)$ satisfies

$$
K^{2}(z)=P(z) k_{1}^{2}+Q(z) k_{2}^{2}
$$

where

$$
P(z)=\frac{N^{2}(z)-n^{2} \omega_{0}^{2}}{n^{2} \omega_{0}^{2}-f^{2}}, \quad Q(z)=P(z)+\frac{n^{2} \omega_{0}^{2} f_{h}^{2}}{\left(n^{2} \omega_{0}^{2}-f^{2}\right)^{2}}
$$

Obviously, (21) denotes the dispersion relation for freely oscillating internal waves. The zero points of $P$ and $Q$ (for constant $N$ ) are

$$
\begin{aligned}
P_{1} & =\min \left[f^{2}, N^{2}\right], \quad P_{2}=\max \left[f^{2}, N^{2}\right] \\
Q_{1,2} & =\frac{N^{2}+f^{2}+f_{h}^{2}}{2}\left[1 \pm \sqrt{1-\frac{4 N^{2} f^{2}}{\left(N^{2}+f^{2}+f_{h}^{2}\right)^{2}}}\right], \quad Q_{1}<Q_{2}
\end{aligned}
$$

First, it is easy to verify that $Q_{1}<P_{1} \leq P_{2}<Q_{2}$, which provides the actual range of each frequency. Furthermore, if assuming $N^{2} \neq f^{2}$, (19) shows that for the form of the surface $\omega_{0}=$ constant in the $\left(k_{1}\right.$, $k_{2}, K$ ) plane over the frequency range $Q_{1}<n^{2} \omega_{0}^{2}<Q_{2}$ there exist three possibilities, as given below:

(1) For $P_{1}<n^{2} \omega_{0}^{2}<P_{2}$, there satisfies $0<P<Q$. $\omega_{0}=$ constant lies on the surface of a cone of elliptical cross-section centered about the $k$-axis (see Fig. $1 \mathrm{a}$ ).

(2) For $P_{2}<n^{2} \omega_{0}^{2}<Q_{2}$, or, $Q_{1}<n^{2} \omega_{0}^{2}<P_{1}$, there satisfies $P<0$ and $Q>0$. $\omega_{0}=$ constant lies on the surface of a cone centered about the $k_{2}$-axis (see Fig. 1c).

(3) if $n^{2} \omega_{0}^{2}=N^{2}$, then $P=0$, the surface of constant $\omega_{0}$ degenerates to a pair of planes (see Fig. $1 \mathrm{~b}$ ).

The above conclusions agree with that proposed by LeBlond and Mysak (1978), who followed a different way. It is worth noting that $P$ equals -1 if $N^{2}=f^{2}$. In this regard, there is only one possibility for the dispersion relation: if $Q_{1}<n^{2} \omega_{0}^{2}<Q_{2}, \omega_{0}=$ constant lies on the surface of a cone centered about the $k_{2}$-axis, provided $n^{2} \omega_{0}^{2} \neq N^{2}$. In order to determine an exact delineation of the spatial structures of internal waves, we should obtain expressions for the water movement in various environmental conditions. 
(a)
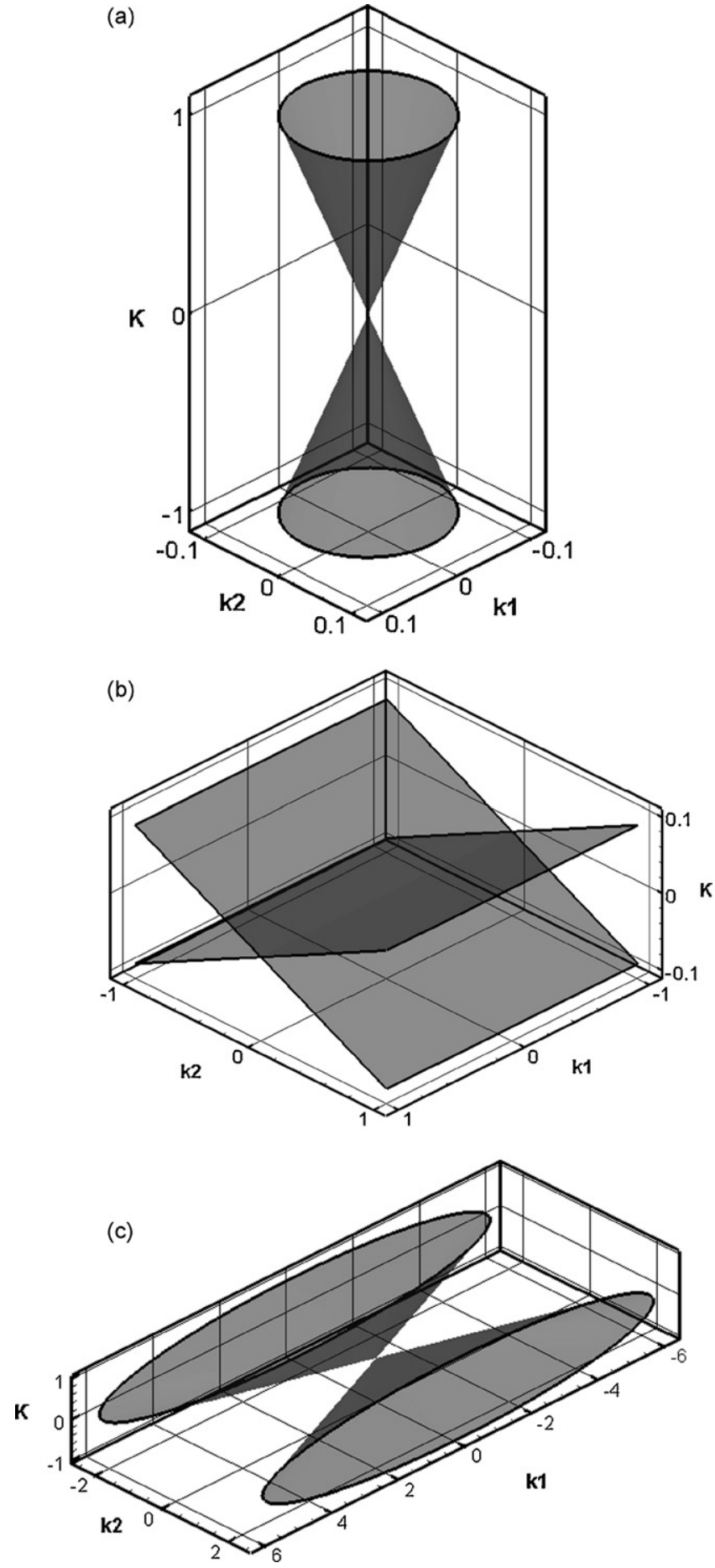

Fig. 1. Contour plots of the dispersion relation. $\theta=30^{\circ}, N=1.4 \times 10^{-3}, \omega_{0}=1.4 \times 10^{-4}$; (a)-(c) correspond to $n=1,10$, and 11 . 


\subsection{Relevance of the non-traditional approximation}

The dispersion relation (21) together with (22) make clear that the non-traditional approximation can become indispensable, or, in other words, the horizontal component of the earth's rotation vector plays a crucial role in the internal tide generation when $P(z)$ is much less than $Q(z)$, especially for the extreme situation $P(z)<0$ is satisfied. This corresponds to two possibilities for the frequency ranges, i.e., the buoyancy frequency $N$ is less than the tidal frequency $\omega_{0}$, whilst $\omega_{0}$ is larger than the vertical component of Coriolis vector $f$; or, $N>\omega_{0}$, whilst $\omega_{0}<f$

The precondition for the second frequency window can become true at the oceans where the latitude is higher than the critical value, as stressed in the introduction. Under this situation, the non-traditional approximation can reasonably explain the activities of internal tides above the critical latitude. In what follows, we point out the practical background for the aforementioned first situation.

According to the traditional theory, freely propagating internal gravity waves (in the layer where $N>f$ ) will be reflected as approaching the layers where $N<f$ and only evanescent waves (amplitudes of which decay exponentially) can exist. However, in deep oceans, stratification can usually be very weak ( $N$ approaches $f$ ) and the horizontal component of the earth's rotation vector becomes more important for the generation of gyroscopic waves. In this situation, the traditional approximation turns to be inapplicable, as was pointed out by LeBlond and Mysak (1978). The limiting wave type for $N \rightarrow 0$ is called pure gyroscopic internal waves. Recently, the existence of gyroscopic waves in deep Mediterranean Sea has been evidenced by Van Haren and Millot (2005). Obviously, the non-traditional approximation has thus been confirmed irreplaceable for elucidating the properties of internal tide in nearly homogeneous deep layers. On the other hand, the weak stratification assumption is in fact not suitable for the whole depth of the ocean and the propagation and transition of internal waves between well-stratified and homogeneous layers are worthy of discussion, but are beyond the scope of the current paper.

\section{Solutions for constant stratification}

For $N(z)$ constant, the general solution of $(20)$ is

$$
\varphi(z)=i n \omega_{0} \frac{\sin K z}{\sin \left(-K h_{0}\right)} \exp \left(\frac{i f f_{h} k_{2}}{n^{2} \omega_{0}^{2}-f^{2}} h_{0}\right)
$$

Substituting the above equation into (17) and (19) and applying the inverse Fourier transform, one obtains

$$
W=\frac{-1}{2 \pi} \sum_{n} i n \omega_{0} e^{i n \omega_{0} \eta} \int_{-\infty}^{\infty} \int_{-\infty}^{\infty} \overline{\bar{H}}\left(k_{1}, k_{2}\right) \frac{\sin K z}{\sin K h_{0}} J_{n}(\kappa) e^{i k_{1} \xi+i k_{2}\left[\zeta+\left(\left(f f_{h}\left(z+h_{0}\right)\right) /\left(n^{2} \omega_{0}^{2}-f^{2}\right)\right)\right]} d k_{1} d k_{2}
$$

For the dispersion relation there exist three possibilities, which thus result in the following three kinds of wave types.

\section{1. $0<P<Q$}

It is desirable to introduce the following transformation:

$$
\begin{aligned}
& \left(\begin{array}{l}
k_{1} \\
k_{2}
\end{array}\right)=k_{r}\left(\begin{array}{c}
\frac{\cos \beta}{\sqrt{P}} \\
\frac{\sin \beta}{\sqrt{Q}}
\end{array}\right), \\
& \left(\begin{array}{l}
\xi \\
\zeta+\frac{f f_{h}\left(z+h_{0}\right)}{n^{2} \omega_{0}^{2}-f^{2}}
\end{array}\right)=r\left(\begin{array}{c}
\sqrt{P} \cos \alpha \\
\sqrt{Q} \sin \alpha
\end{array}\right), \quad r>0, \quad 0 \leq \alpha<2 \pi, \quad-\infty<k_{r}<\infty, \quad 0 \leq \beta \leq \pi
\end{aligned}
$$


(25) turns to

$$
W=-\sum_{n=-n_{0}}^{n_{0}} \frac{i n \omega_{0}}{2 \pi} e^{i n \omega_{0} \eta} \int_{0}^{\pi} \int_{-\infty}^{\infty} \frac{\overline{\bar{H}}\left(k_{r}, \beta\right)}{\sqrt{P Q}} \frac{\sin K z}{\sin K h_{0}} J_{n}(\kappa) e^{i k_{r} r \Theta(\beta)}\left|k_{r}\right| d k_{r} d \beta
$$

where

$$
\Theta(\beta)=\cos (\beta-\alpha), \quad K=\left|k_{r}\right|
$$

Here, the tidal excursion parameter

$$
\kappa=\frac{k_{r}}{\omega_{0}}\left(\frac{\cos \beta}{\sqrt{P}} U_{0}+\frac{\sin \beta}{\sqrt{Q}} V_{0}\right)
$$

satisfies

$$
\kappa\left(k_{r}, \beta+\pi\right)=\kappa\left(-k_{r}, \beta\right)=-\kappa\left(k_{r}, \beta\right)
$$
$k$-axis:

One can readily identify that for $K>0$, the poles of the integrand involved in (27) lie on the real

$$
\left|k_{r m}\right|=\frac{m \pi}{h_{0}}, \quad m=1,2,3, \ldots
$$

To estimate the inner integrand of (27), Lighthill's method (1978) is employed which suggests using $\omega_{0}-i \varepsilon$ to replace frequency $\omega_{0}$. Then, $\exp \left(i \omega_{0} t\right)$ is replaced by $\exp \left(\varepsilon t+i \omega_{0} t\right)$, giving the source a slow exponential growth from zero at $t=-\infty$ to its present level. Looking exclusively for the associated growing waves, varying with time like $\exp \left(\varepsilon t+i \omega_{0} t\right)$, the danger of the solution being contaminated by other wave energy generated 'at infinity' is then excluded. (Here, $\varepsilon$ is a small positive number which is later allowed to tend to zero.) Thus, in keeping with Cauchy's theorem, for $r \Theta(\beta)>0$, raising the poles with a distance $k^{\prime}\left(k^{\prime}\right.$ tends to zero when let $\varepsilon \rightarrow 0$ ), giving a contribution of $(+2 \pi i)$ times the residuum from each. Similarly, for $r \Theta(\beta)<0$, lowering the poles with a distance $k^{\prime}$, giving a contribution of $(-2 \pi \mathrm{i})$ times the residuum from each.

Furthermore, from the dispersion relation (21), it is not difficult to derive that the group velocities along $\left(k_{r}, \beta\right)$ are

$$
\left(\left.\frac{\partial \omega}{\partial k_{r}}\right|_{\omega=n \omega_{0}},\left.\frac{\partial \omega}{\partial \beta}\right|_{\omega=n \omega_{0}}\right)=\varpi\left(\frac{k_{r}}{n \omega_{0}}, 0\right)
$$

where

$$
\varpi=\frac{\left(n^{2} \omega_{0}^{2}-f^{2}\right)^{3}}{\left(n^{2} \omega_{0}^{2}-f^{2}\right)^{2}\left(k_{1}^{2}+k_{2}^{2}+K^{2}\right)+k_{2}^{2} f^{2} f_{h}^{2}}
$$

This indicates that $\operatorname{sgn}\left(\partial \omega / \partial k_{r}\right)=\operatorname{sgn}\left(n k_{r}\right)$ provided that $n^{2} \omega_{0}^{2}>f^{2}$. For simplicity, we only present the case of $n^{2} \omega_{0}^{2}>f^{2}$ and that for $n^{2} \omega_{0}^{2}<f^{2}$ can be derived in a similar way.

For $r \Theta(\beta)>0$ and $n>0$, only the poles, $-k_{r m}$, are moved over the axis with a distance $i k^{\prime}\left(k^{\prime}=\varepsilon n \omega_{0} / \varpi k_{r m}\right)$, provided that $\omega_{0}$ is raised by an increment $-i \varepsilon$. Similarly, for $r \Theta(\beta)>0$ and $n<0$, if $\omega_{0}$ is increased by an increment $i \varepsilon$, only the poles, $k_{r m}$, are moved over the axis with a distance $i k^{\prime}$. As the limit $\varepsilon \rightarrow 0$ is approached, the inner integral looks like

$$
\begin{aligned}
W= & \sum_{m=1}^{\infty} \frac{2 m \pi}{h_{0}^{2}} \sin \left(\frac{m \pi}{h_{0}} z\right) \sum_{n=1}^{n_{0}}(-1)^{m+n+1} \frac{n \omega_{0}}{\sqrt{P Q}} \int_{0}^{\pi} \overline{\bar{H}}\left(k_{r m}, \beta\right) \\
& \times J_{n}\left[\kappa\left(k_{r m}, \beta\right)\right] \cos \left[k_{r m} r \Theta(\beta)-n \omega_{0} \eta\right] d \beta
\end{aligned}
$$


Similarly, for $r \Theta(\beta)<0$, the solution reads

$$
\begin{aligned}
W= & \sum_{m=1}^{\infty}(-1)^{m+1} \frac{2 m \pi}{h_{0}{ }^{2}} \sin \left(\frac{m \pi}{h_{0}} z\right) \sum_{n=1}^{n_{0}} \frac{n \omega_{0}}{\sqrt{P Q}} \int_{0}^{\pi} \overline{\bar{H}}\left(k_{r m}, \beta\right) \\
& \times J_{n}\left[\kappa\left(k_{r m}, \beta\right)\right] \cos \left[k_{r m} r \Theta(\beta)+n \omega_{0} \eta\right] d \beta
\end{aligned}
$$

where we have made use of the relationship:

$$
J_{-n}(x)=J_{n}(-x)=(-1)^{n} J(x)
$$

Suppose the bottom function is written as

$$
H(x, y)=F(x) F(y)
$$

Then $F(y)=1$ represents the solution in the one dimension limit. In this limit, the solution of (33) and (34) reduces to:

$$
W(\xi, z, t)=\left\{\begin{array}{l}
\frac{-2 \sqrt{2}}{m \sqrt{\pi}} \sum_{n=1}^{\infty} \sum_{m=1}^{\infty}(-1)^{m+n} k_{m} n \omega_{0} J_{n}\left(\frac{U_{0} k_{m}}{\omega_{0}}\right) \sin \frac{m \pi z}{h_{0}} \times \bar{H}\left(k_{m}\right) \cos \left(k_{m} \xi-n \omega_{0} t\right), \quad \xi>0 \\
\frac{-2 \sqrt{2}}{m \sqrt{\pi}} \sum_{n=1}^{\infty} \sum_{m=1}^{\infty}(-1)^{m} k_{m} n \omega_{0} J_{n}\left(\frac{U_{0} k_{m}}{\omega_{0}}\right) \sin \frac{m \pi z}{h_{0}} \bar{H}\left(k_{m}\right) \cos \left(k_{m} \xi+n \omega_{0} t\right), \quad \xi<0
\end{array}\right.
$$

Abandoning the non-traditional approximation, namely, removing the horizontal components of the Coliolis vector in (1), the above solution is consistent with Khatiwala's solution (Khatiwala, 2003).Furthermore, when $r \rightarrow \infty$, the integrand in (33) has stationary points $\beta=\alpha$ (for $0<\alpha<\pi$ ), or $\beta=\alpha-\pi$ (for $\pi<\alpha<2 \pi$ ), satisfying $\partial \Theta(\beta) / \partial \beta=0$. Ultimately, the stationary phase approximation (see, for instance, Nayfeh, 1973) yields:

$$
\begin{aligned}
W(r, \alpha, z, \eta)= & \sum_{m=1}^{\infty} \sum_{n=1}^{n_{0}}(-1)^{m+n+1} \frac{2 \pi}{h_{0}^{3 / 2}} \sqrt{\frac{2 m}{r}} \frac{n \omega_{0}}{\sqrt{P Q}} \sin \left(\frac{m \pi}{h_{0}} z\right) \overline{\bar{H}}\left(k_{r m}, \alpha\right) \\
& \times J_{n}\left[\kappa\left(k_{r m}, \alpha\right)\right] \cos \left[k_{r m} r-n \omega_{0} \eta-\frac{\pi}{4}\right]
\end{aligned}
$$

where we have assumed that

$$
\overline{\bar{H}}\left(k_{r}, \alpha-\pi\right)=\overline{\bar{H}}\left(k_{r}, \alpha\right)
$$

4.2. $P<0, Q>0$

In this case, (25) can be simplified with help of the following transformations:

$$
\begin{aligned}
& \left(\begin{array}{l}
k_{1} \\
k_{2}
\end{array}\right)=k_{r}\left(\begin{array}{c}
\frac{\cos \beta}{\sqrt{-P}} \\
\frac{\sin \beta}{\sqrt{Q}}
\end{array}\right), \\
& \left(\begin{array}{c}
\xi \\
\zeta+\frac{f f_{2}\left(z+h_{0}\right)}{n^{2} \omega_{0}^{2}-f^{2}}
\end{array}\right)=r\left(\begin{array}{c}
\sqrt{-P} \cos \alpha \\
\sqrt{Q} \sin \alpha
\end{array}\right), \quad r>0, \quad 0 \leq \alpha<2 \pi, \quad-\infty<k_{r}<\infty, \quad 0 \leq \beta \leq \pi
\end{aligned}
$$

A notable distinction of the appearance of wave field comparing with the former case is that propagation of the internal waves is now confined in the area surrounded by two lines, $P k_{1}^{2}+Q k_{2}^{2}=0$, 
namely:

$$
\left|\frac{k_{2}}{k_{1}}\right|>\sqrt{-\frac{P}{Q}}
$$

After a lengthy and similar derivation, the final result for the wave filed, as $r \rightarrow \infty$, can be given asymptotically by:

$$
\begin{aligned}
W(r, \alpha, z, \eta)= & \sum_{m=1}^{\infty} \sum_{n=n_{1}}^{n_{2}}(-1)^{m+n+1} \frac{2 \pi}{h_{0}^{3 / 2}} \sqrt{\frac{2 m}{r}} \frac{n \omega_{0}}{\sqrt{-P Q}} \sin \left(\frac{m \pi}{h_{0}} z\right) \frac{\overline{\bar{H}}\left(k_{r m},-\alpha\right)}{(-\cos 2 \alpha)^{1 / 4}} \\
& \times J_{n}\left[\kappa\left(k_{r m},-\alpha\right)\right] \cos \left[\frac{m \pi}{h_{0}} r \sqrt{-\cos 2 \alpha}-n \omega_{0} \eta-\frac{\pi}{4}\right]
\end{aligned}
$$

\section{3. $P=0$}

In this case, the original (3D) model degenerates to a 2D one and internal waves travel in two $2 \mathrm{D}$ planes. As this article is chiefly focused on the 3D situation the 2D case that has been discussed exhaustively by previous studies is hence omitted in this text.

\subsection{Demarcation of wave types}

Before visualizing and examining wave structures, it is necessary to present a proper demarcation of wave types. The most straightforward way is to estimate the sign of parameter $P$, as discussed previously. On the other hand, in the previously reported 2D investigations the wave types are well classified by analyzing the directions of internal wave rays (Fan and Singh, 2008), which, however, can be proved insufficient for the classification of wave types in the 3D space. Actually, the 3D problem is complicated in that internal waves may have different shapes in the horizontal plane even if the rays keep the same angles as those in the $2 \mathrm{D}$ case. To properly distinguish the various wave types, the method used by LeBlond and Mysak (1978) for analyzing the dispersion relation can be applied (see Appendix B).

It is found that the wave types can be demarcated by three limitations of the frequency, namely, $n^{2} \omega_{0}^{2}, N_{c}{ }^{2}$ and $f^{2}$ (see Fig. 2 and Table 1 ). Specifically, the types that are generated below the critical latitude $\left(n^{2} \omega_{0}^{2}-f_{2}>0\right)$ are (a), (d), (e) and (f), where the buoyancy frequency satisfies $N^{2}>n^{2} \omega_{0}^{2}$,

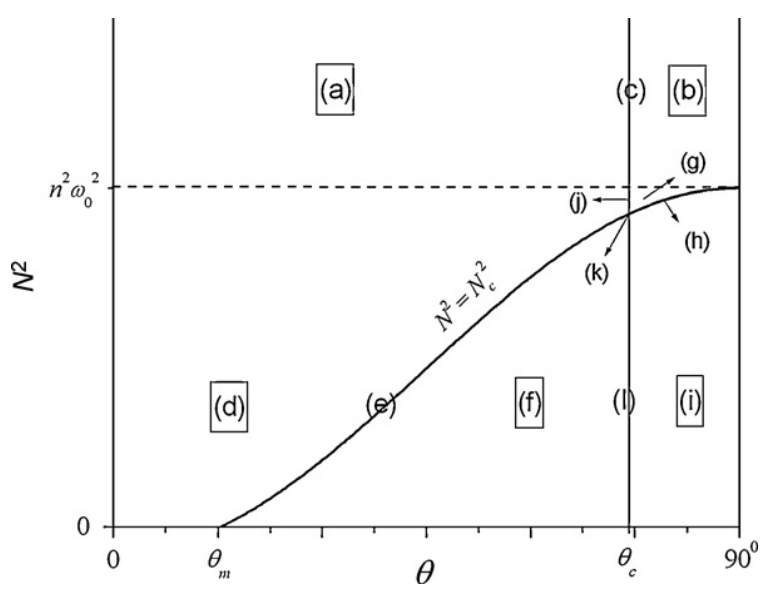

Fig. 2. Illustration for the demarcation of wave types. (a)-(1) corresponds to each wave type that is depicted in Table 1. 
Table 1

Twelve kinds of typical wave types corresponding to each possibility of frequency ranges.

\begin{tabular}{|c|c|c|c|c|c|}
\hline \multirow[t]{2}{*}{ Wave types } & \multicolumn{4}{|c|}{ Ranges of buoyancy frequency } & \\
\hline & $N^{2}>n^{2} \omega_{0}^{2}$ & $N_{c}^{2}<N^{2}<n^{2} \omega_{0}^{2}$ & $N^{2}=N_{c}^{2}$ & $N^{2}<N_{c}^{2}$ & \\
\hline \multicolumn{6}{|c|}{ Ranges of latitudes } \\
\hline & (a) & (d) & (e) & (f) & \\
\hline \multirow{3}{*}{$n^{2} \omega_{0}^{2}>f^{2}$} & OP & OP & OEOV & EW & $\mathrm{RD}$ \\
\hline & $\mathrm{EL}$ & $\mathrm{HL}$ & $\mathrm{HL}$ & $\mathrm{HL}$ & $\mathrm{PH}$ \\
\hline & (b) & (g) & (h) & (i) & \\
\hline \multirow[t]{3}{*}{$n^{2} \omega_{0}^{2}<f^{2}$} & PW & PW & OPOV & $\mathrm{OP}$ & $\mathrm{RD}$ \\
\hline & $\mathrm{HL}$ & EL & $\mathrm{EL}$ & EL & $\mathrm{PH}$ \\
\hline & (c) & (j) & $(\mathrm{k})$ & (1) & \\
\hline \multirow{2}{*}{$n^{2} \omega_{0}^{2}=f^{2}$} & OHOP & OHOP & $\mathrm{OHOV}$ & OHOE & $\mathrm{RD}$ \\
\hline & $\mathrm{OHL}$ & $\mathrm{OHL}$ & $\mathrm{OHL}$ & $\mathrm{OHL}$ & $\mathrm{PH}$ \\
\hline
\end{tabular}

Note here RD means ray directions with respect to vertical; PH means projection in horizontal plane; OP means opposite; OEOV means one equator-ward and the other vertical; OPOV means one poleward and the other vertical; OHOP means one horizontal and the other poleward; OHOV means one horizontal and the other vertical; OHOE means one horizontal and the other equatorward; EW means both equator-ward; PW means both poleward; EL means ellipse; HL means hyperbolic lines; OHL means one branch of hyperbolic lines.

$N_{c}^{2}<N^{2}<n^{2} \omega_{0}^{2}, N_{2}=N_{c}^{2}$; and $N_{2}<N_{c}^{2}$, respectively. In the same ranges of buoyancy frequency, the wave types (c), (j), (k), and (l) correspond to those generated at the critical latitude, where $n^{2} \omega_{0}^{2}-f^{2}=$ 0 ; and (b), (g), (h), and (i) to those created above the critical latitude $\left(n^{2} \omega_{0}^{2}-f^{2}<0\right)$. Comparing (b) and $(\mathrm{g})$, one can find a common feature that the rays are both poleward in both cases. However, in the horizontal plane the wave shapes are quite different. This prominent characteristic that arises for the 3D internal wave structures cannot be distinguished with a 2D case.

\subsection{Examples for visualizations of solutions}

Before commenting on the former solutions for wave fields, it should be mentioned that unlike the complicated ring type topography discussed in Bühler and Muller (2007) we focus entirely in this paper on a single Gaussian hump, defined by:

$$
H_{2}\left(x_{s}, y_{s}\right)=H_{0} e^{-\left(x_{s} / a\right)^{2} / 2-\left(y_{s} / b\right)^{2} / 2}
$$

where $\left(x_{s}, y_{s}\right)$ denotes the major axis of the topography. It should be stated that such a single Gaussian topography is, though, simpler but is sufficient to investigate the abnormality of non-traditional properties of internal tides.

\subsection{1. (a) $P>0$}

As a first step, cases at the lower latitude are examined, where parameters are $\theta=30^{\circ}, N=1.4 \times 10^{-3}$, $\omega_{0}=1.4 \times 10^{-4}, n=1, a=b=10^{4} \mathrm{~m}, U_{0}=0, V_{0}=0.01 \mathrm{~m} / \mathrm{s}, h_{0}=5000 \mathrm{~m}$, and $H_{0}=500 \mathrm{~m}$, which fall into the effective range of a real deep ocean (St. Laurent and Garrett, 2002). For these parameters the excursion parameter, $\kappa \sim O\left(V_{0} / a \omega_{0}\right)=10^{-2}$, is much less than 1 . Thus, the term for $n=1$, the lowest harmonic, dominates the Bessel function in (38) (St. Laurent and Garrett, 2002; Garrett and Kunze, 2007). As a consequence, we merely need to examine the situation for $n=1$ in the ensuing discussion. Parameters, $P$ and $Q$ involved in the dispersion relation (21) are, respectively, 135.6 and 137.1, which indicates the wave types belong to that discussed in Section 4.1.

Fig. 3. The two-dimension plots of the wave fields in the $x-y$ plane $\left(z=-h_{0} / 2\right)$. The common parameters in (a) $-(\mathrm{c})$ are $\theta=30^{\circ}$, $N=1.4 \times 10^{-3}, \omega_{0}=1.4 \times 10^{-4}, \rho_{0}=10^{3} \mathrm{~kg} / \mathrm{m}^{3}, n=1, h_{0}=5000 \mathrm{~m}$, and $H_{0}=500 \mathrm{~m}$. The tidal current conditions are, in turns, $U_{0}=0$, $V_{0}=0.01 \mathrm{~m} / \mathrm{s}$ (in Fig. 3a and c) and $U_{0}=V_{0}=0.01 \mathrm{~m} / \mathrm{s}$ (in Fig. $3 \mathrm{~b}$ ). Lengths of the major axes of the bottom topography are $a=b=10^{4} \mathrm{~m}$ (in Fig. 3a and b) and $a=2 \times 10^{4} \mathrm{~m}$ and $b=0.5 \times 10^{4} \mathrm{~m}$ (in Fig. 3c). To facilitate the visualization, the horizontal coordinate is normalized, hereinafter, by the total horizontal length $Y=200 \mathrm{~km}$. 

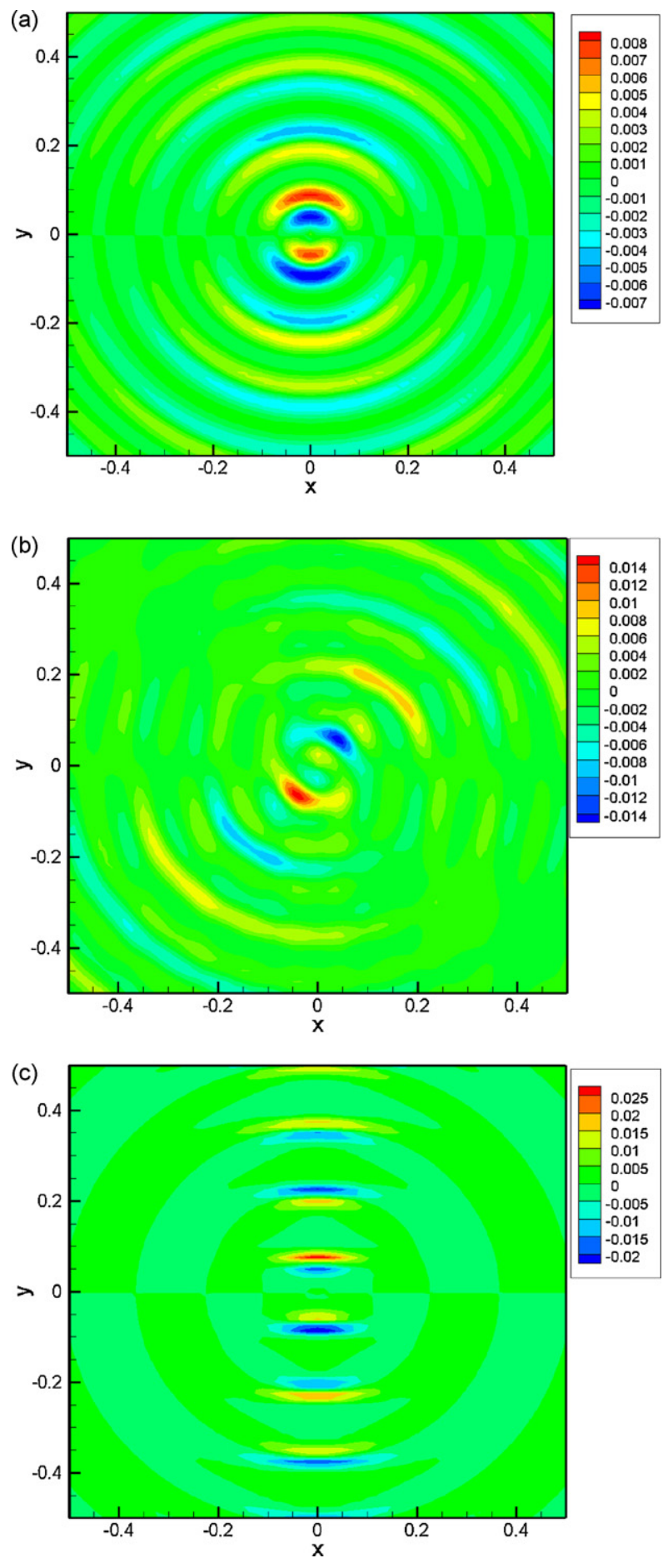

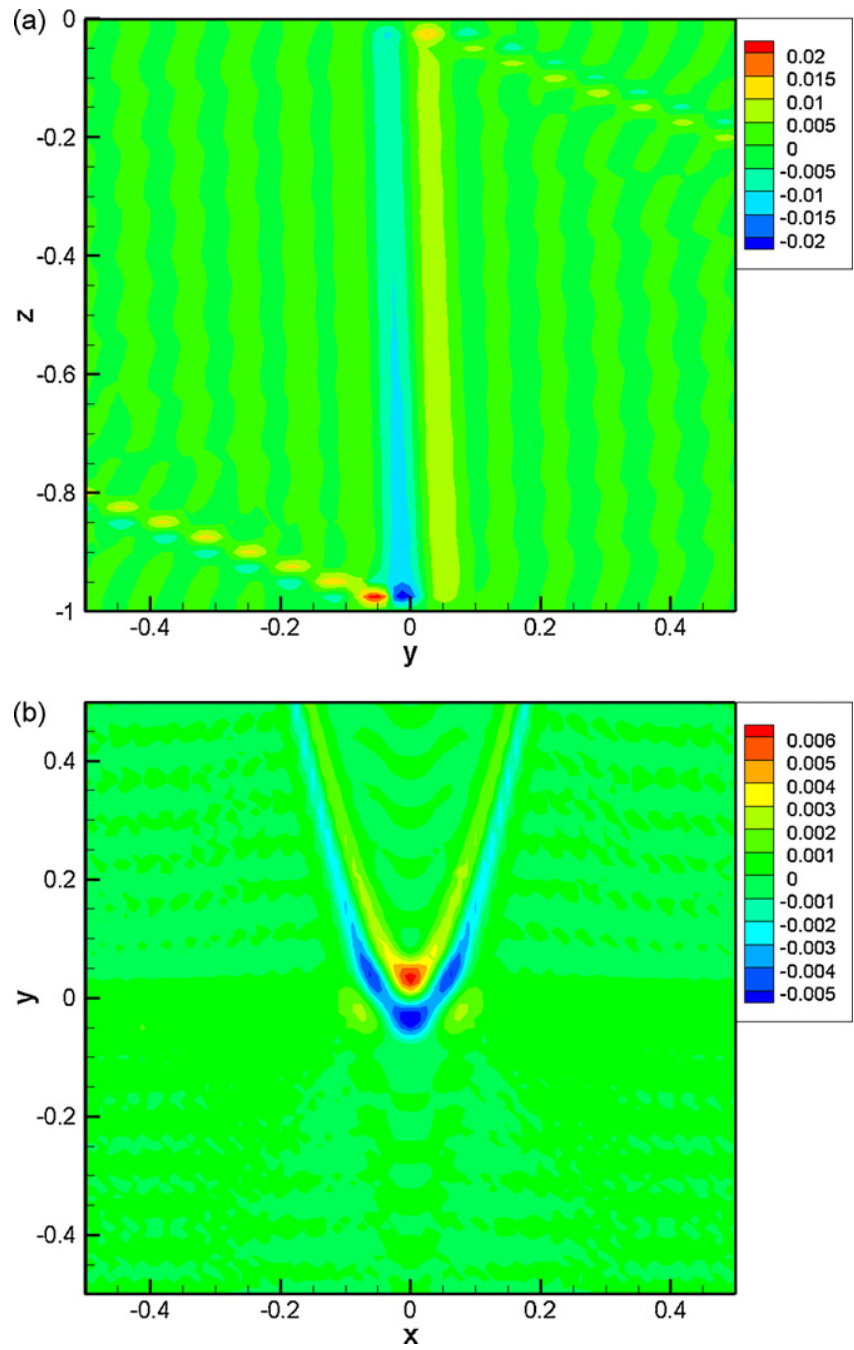

Fig. 4. Contour plots of the buoyancy field in the $x-z$ plane where $y=0$ (a), and in the $x-y$ plane where $z=-h_{0} / 2$ (b). Comparing to Fig. 3(a) different parameters are: $\theta=74^{\circ}, N=0.4 \times 10^{-4}$ and $U_{0}=0.01, V_{0}=0 \mathrm{~m} / \mathrm{s}$.

Fig. 3(a) is a 2D plot in the $x-y$ plane at half the total depth $z=-h_{0} / 2$, where the characteristic of plane waves is clear. The waves of constant phase are nearly in circles, mostly attributed to the fact that parameters $P$ and $Q$ for dispersion relation are approximately identical. Besides, another phenomenon is prominent and deserves attention. The waves are intensified in the $y$-direction (the direction of tidal flow). This is likely attributed to the consideration of the basic tidal flow retained in the governing equation (1). The effect of superposition of the basic flow forces the propagation of internal waves in the tidal direction much more than in other directions. This shows that the effect of the basic flow is not only the reason for the occurrence of higher harmonics as proposed first by Bell (1975a) but also enhances the wave strength in the direction of basic flow.

Besides, tidal flow in different direction is considered and wave fields are plotted in Fig. 3(b) where the direction of tidal flow is changed by $45^{\circ}$. It is seen in Fig. 3(b) that the constant wave phases that have been strengthened by the basic tidal flow are inclined with respect to the $x$-direction at an angle $45^{\circ}$, which is just the direction of the tidal flow. The influence of asymmetry of the bottom profile is 

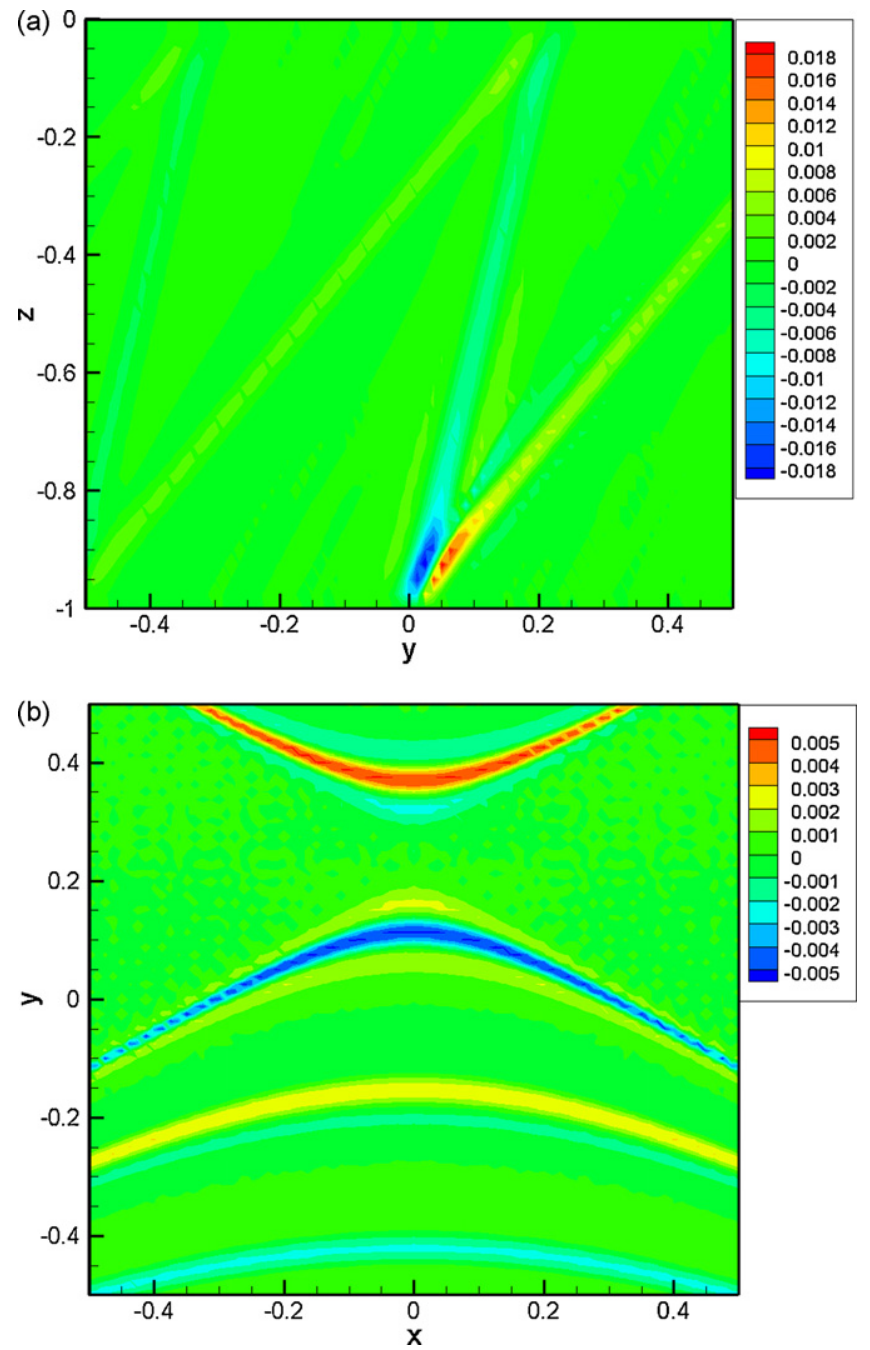

Fig. 5. Contour plots of the buoyancy field in the $x-z$ plane where $y=0$ (a), and in the $x-y$ plane where $z=-h_{0} / 2$ (b). Comparing to Fig. 3(a) different parameters are: $\theta=75^{\circ}, N=4 \times 10^{-4}$.

illustrated in Fig. 3(c) where the lengths of major axes of topography are changed to $a=2 \times 10^{4} \mathrm{~m}$ and $b=0.5 \times 10^{4} \mathrm{~m}$. The waves of constant phases are not in circles any longer, but in thick and short arcs parallel in the $x$-direction, showing that the 2D characteristics of internal waves emerge due to the increased length ratio between the major axes of topography.

\subsection{2. (b) $P<0$}

In the above discussion, parameter $P$ is positive and the appearance of constant phase planes look like circles, straight lines, or ellipses. Besides these easily understood wave shapes, the wave characteristics for $P$ less than zero are also noteworthy and need to be examined. Fig. 4 depict internal wave generations at latitude $\theta=74^{\circ}$, with the parameter different from Fig. 3, as $N=0.4 \times 10^{-4}$ $\left(<N_{c}=1.34 \times 10^{-4}\right)$. Fig. $4(\mathrm{a})$ plots the buoyancy field in the $x-z$ plane $(y=0)$, where the pair of internal wave rays appear equator-ward. This generates a hyperbolic constant phase plane, as illustrated in Fig. 4(b). Furthermore, according to (22), values for $P$ and $Q$ are -338.3 and 10,789, respectively. 
Substituting into (41), one can find that slopes of degenerate lines are $|\tan \gamma|=k_{1} / k_{2}=(-Q / P)^{1 / 2}=32$, which are in agreement with what has been shown in Fig. 4(b).

Another case for $P<0$ is shown in Fig. 5, where the latitude $\theta=75^{\circ}\left(>\theta_{c}\right)$ and the buoyancy frequency $N=4 \times 10^{-4}\left(>\omega_{0}\right)$. Accordingly, the values for $P$ and $Q$ are -1025.3 and 455.8 , respectively. In contrast to Fig. 4(a), the directions of internal beams are utterly different, which are both poleward. The differences between ray slopes are not great, which lead to the appearance of two branches of the hyperbolic constant phase lines, as illustrated in Fig. 5(b). Note that the slopes of degenerate lines here are $|\tan \gamma|=0.44$, much less than those in Fig. 4(b).

\section{Summary and conclusions}

In this paper the model of internal tide generation over two-dimensional topographies is described, in which the horizontal components of the earth's rotation are retained and the effect of basic flow is taken into consideration. Unlike previous studies focusing primarily on the tidal energy conversion, the major objective of this paper is to provide an overall and a detailed description for the wave structures under various parameter conditions. For this purpose, Lighthill's method is employed to derive the resulting wave fields. For a homogeneous ocean, the wave configurations can have, basically, three kinds of projections on the horizontal plane: the ellipse, hyperbolic lines, and a pair of lines. This result is easily understood from the known conformation of the dispersion relations. Besides this fundamental understanding of the internal wave structures, more prototypes of wave structures are identified and demarcated by scrutinizing the relationship between the cone angle of the wave number surface and the angle formed by the axes of the cone and the earth's Cartesian coordinates.

The various possible wave types can be classified specifically into 12 kinds by comparing the magnitudes of 4 frequency types: the buoyancy frequency, $N$, and its critical value, $N_{c}$, the wave frequencies, $n \omega_{0}$, and the vertical component of the earth's rotation, $f$. This finding shows a remarkable contrast to the previous 2D results, in which five kinds of modes for internal beams are identified. In a 2D theory, the vertical structures of internal waves with $N>N_{c}$ display a common characteristic that internal wave rays are in opposite directions (or both poleward) with respect to the vertical, provided $n \omega_{0}>f\left(n \omega_{0}<f\right.$ ). However, the difference of wave patterns on the horizontal plane cannot be distinguished, unless a 3D theory is implemented. In fact, the waves with $N>n \omega_{0}$ show ellipses on the horizontal plane below the critical value, whilst those with $N<n \omega_{0}$ show arcs of hyperbolic lines, although for both $N>N_{c}$.

Finally, the effect of basic tidal flow is found to be twofold. As suggested by the previous investigations, not only the internal waves of the basic tidal frequency but also the higher harmonics can be generated if the basic tidal current is taken into consideration. On the other hand, the wave strength gets amplified in the tidal direction as a consequence of the superimposed effect of the tidal current.

\section{Acknowledgment}

This work was supported by the National Natural Science Foundation of China under Grant 10602060.

\section{Appendix A. Fourier representation of bottom topography in the polar coordinates}

If the bottom function in the coordinates of the major axis of the topography $\left(x_{s}, y_{s}\right)$ can be expressed as:

$$
H_{2}\left(x_{s}, y_{s}\right)=H_{0} e^{-\left(x_{s} / a\right)^{2} / 2-\left(y_{s} / b\right)^{2} / 2}
$$

where subscript $s$ denotes the quantities in the coordinates of the major axis of the topography, then the Fourier transform of the bottom function in the coordinate system $\left(k_{r}, \beta\right)$ can be written as:

$$
\overline{\bar{H}}\left(k_{r}, \beta\right)=H_{0} a b e^{-\left(k_{r}^{2} / 2\right)\left[a^{2}(\cos \chi(\cos \beta / \sqrt{P})+\sin \chi(\sin \beta / \sqrt{Q}))^{2}+b^{2}(\cos \chi(\sin \beta \sqrt{\bar{Q}})-\sin \chi(\cos \beta / \sqrt{P}))^{2}\right]}
$$


where $\chi$ is the angle between the coordinates $(x, y)$ and $\left(x_{s}, y_{s}\right)$, satisfying

$$
\left(\begin{array}{l}
x_{s} \\
y_{s}
\end{array}\right)=\left(\begin{array}{l}
\cos \chi, \sin \chi \\
-\sin \chi, \cos \chi
\end{array}\right)\left(\begin{array}{l}
x \\
y
\end{array}\right)
$$

\section{Appendix B. Explanations for the wave type demarcation}

Let us go back to (18), the basic equation for the vertical movement of water whose periodic wave solution can be assumed as:

$$
w_{n}=W_{n} \cdot e^{i\left(k_{1} \xi+k_{2} \zeta+k_{3} z-n \omega_{0} \eta\right)}
$$

Substitution of (47) into (18) yields the dispersion relation:

$$
\left(N^{2}-n^{2} \omega_{0}^{2}\right) k_{1}{ }^{2}+\left(N^{2}-n^{2} \omega_{0}^{2}+f_{h}{ }^{2}\right) k_{2}{ }^{2}+2 f f_{h} k_{2} k_{3}-\left(n^{2} \omega_{0}^{2}-f^{2}\right) k_{3}{ }^{2}=0
$$

To eliminate the cross-term, LeBlond and Mysak (1978) introduced a rotating transform, defined as:

$$
\left(\begin{array}{l}
k_{1} \\
k_{2} \\
k_{3}
\end{array}\right)=\left(\begin{array}{lll}
1 & 0 & 0 \\
0 & \cos v & -\sin v \\
0 & \sin v & \cos v
\end{array}\right)\left(\begin{array}{l}
K_{1} \\
K_{2} \\
K_{3}
\end{array}\right)
$$

where the angle of rotation $v$ about the $\xi$-axis is given as:

$$
\tan 2 v=\frac{2 f f_{h}}{N^{2}-f^{2}+f_{h}{ }^{2}}=\frac{\sin 2 \theta}{N^{2} / 4 \Omega^{2}+\cos 2 \theta}
$$

Applying (49), the dispersion relation (48) takes on a more concise and symmetric form:

$$
\left(N^{2}-n^{2} \omega_{0}^{2}\right) K_{1}^{2}+\left(\omega_{a}^{2}-n^{2} \omega_{0}^{2}\right) K_{2}^{2}+\left(\omega_{b}^{2}-n^{2} \omega_{0}^{2}\right) K_{3}^{2}=0
$$

where

$$
\begin{aligned}
& \omega_{a}^{2}=f\left(f+f_{h} \cot v\right)=Q_{2}, \\
& \omega_{b}^{2}=f\left(f-f_{h} \tan v\right)=Q_{1}
\end{aligned}
$$

Note $Q_{1}$ and $Q_{2}$ are two roots of $Q$ [see (23)]. With the assumption of $0<\nu<\pi / 2$ it may be shown that $\omega_{a}$ and $\omega_{b}$ are the upper and lower limits for wave frequencies, namely, $w_{a}^{2}>\left(N^{2}, n^{2} \omega_{0}^{2}, f^{2}\right)>\omega_{b}^{2}$.

The emphasis of this study is aimed at an exact elucidation of the relation between cone angles $\gamma_{w}$ (the angle between the wave number cone and axis $k_{2}$ in the $y-z$ plane) and $\gamma_{g}$ (the angle between the group-velocity cone and axis $k_{2}$ in the $y-z$ plane) and the tilt angle $v$, which is found to be the most efficient method for the demarcation of wave types. From (51), one can readily find that for $N^{2}>n^{2} \omega_{0}^{2}$ the cone angle of the wave number cone, $\gamma_{w}$, is

$$
\tan ^{2} \gamma_{w}=\frac{n^{2} \omega_{0}^{2}-\omega_{b}^{2}}{\omega_{a}^{2}-n^{2} \omega_{0}^{2}}
$$

The group-velocity cone is centered on the $k_{3}$-axis as well and is perpendicular to the wave number cone. Thus, the cone angle of the group-velocity cone, $\gamma_{g}$, satisfies $\gamma_{g}=90^{\circ}-\gamma_{w}$, or

$$
\tan ^{2} \gamma_{g}=\cot ^{2} \gamma_{w}=\frac{\omega_{a}^{2}-n^{2} \omega_{0}^{2}}{n^{2} \omega_{0}^{2}-\omega_{b}^{2}}
$$

Substituting (52) into (53) yields

$$
\tan ^{2} \gamma_{w}=\tan ^{2} v \frac{f f_{h}+\left(n^{2} \omega_{0}^{2}-f^{2}\right) \cot v}{f f_{h}-\left(n^{2} \omega_{0}^{2}-f^{2}\right) \tan v}
$$

Similarly, (54) can be rewritten as:

$$
\tan ^{2} \gamma_{g}=\tan ^{2} v \frac{n^{2} \omega_{0}^{2}-f^{2}+\left(N^{2}-n^{2} \omega_{0}^{2}+f_{h}^{2}\right) \cot ^{2} v}{n^{2} \omega_{0}^{2}-f^{2}+\left(N^{2}-n^{2} \omega_{0}^{2}+f_{h}^{2}\right) \tan ^{2} v}
$$


From (55), it is clear that

$$
\left\{\begin{array}{l}
\gamma_{w}=90^{\circ}-\gamma_{g} \geq v, \quad \text { if } \quad n^{2} \omega_{0}^{2}-f^{2} \geq 0 ; \\
\gamma_{w}=90^{\circ}-\gamma_{g}<v, \quad \text { if } \quad n^{2} \omega_{0}^{2}-f^{2}<0
\end{array}\right.
$$

Furthermore, it is not difficult to prove from (56) that if $N^{2} \neq N_{l}^{2}$ there satisfies

$$
\begin{cases}\gamma_{g} \geq v, & \text { if } \quad N^{2} \geq N_{c}^{2} \\ \gamma_{g}<v, & \text { if } \quad N^{2}<N_{c}^{2}\end{cases}
$$

where $N_{c}^{2}=n^{2} \omega_{0}^{2}-f_{h}^{2}$ and $N_{l}^{2}=f^{2}-f_{h}^{2}$. Particularly, if $N^{2}=N_{l}^{2}$, one can recognize from (50) that $\nu=45^{\circ}$ and (56) is thus inapplicable. In this regard, the appropriate result for $\gamma_{g}$ can be found by substituting $v=45^{\circ}$ into (52) and (54), which hence leads to

$$
\tan ^{2} \gamma_{g}=\frac{f f_{h}-\left(n^{2} \omega_{0}^{2}-f^{2}\right)}{f f_{h}+\left(n^{2} \omega_{0}^{2}-f^{2}\right)}
$$

(59) clearly shows that

$$
\begin{cases}\gamma_{g}>v=45^{\circ}, & \text { if } \quad n^{2} \omega_{0}^{2}-f^{2}<0 \\ \gamma_{g}<v=45^{\circ}, & \text { if } \quad n^{2} \omega_{0}^{2}-f^{2}>0\end{cases}
$$

In general, the wave patterns formed in various ray angles can be deduced from the above relationship between $\gamma_{g}$ and $\nu$. For example, let us consider the case of $N^{2}>n^{2} \omega_{0}^{2}$ and $n^{2} \omega_{0}^{2}-f^{2}>0$. This leads directly to $90^{\circ}-\gamma_{g}>v$, as indicated clearly from (57). Moreover, (58) states that $\gamma_{g}>v$ as $N^{2}>n^{2} \omega_{0}^{2}>N_{c}^{2}$. This therefore shows that in the original earth's Cartesian $(y, z)$ plane the rays emanate in opposite directions with respect to the vertical direction. The wave pattern that is projected on the horizontal plane is as ellipsoidal.

\section{References}

Balmforth, N.J., Ierley, G.R., Young, W.R., 2002. Tidal conversion by subcritical topography. J. Phys. Oceanogr. 32, $2900-2914$.

Bell, T.H., 1975a. Lee waves in stratified flows with simple harmonic time dependence. J. Fluid Mech. 67, 705-722.

Bell, T.H., 1975b. Topographically generated internal waves in the open ocean. J. Geophys. Res. 80, 320-327.

Bühler, O., Muller, C.J., 2007. Instability and focusing of internal tides in the deep ocean. J. Fluid Mech. 588, 1-28.

Durran, D.R., Bretherton, C., 2004. Comments on "The roles of the horizontal component of the earth's angular velocity in non-hydrostatic linear models". J. Atmos. Sci. 61, 1982-1986.

Fan, P., Singh, V.P., 2008. The limiting states of internal wave rays. J. Geophys. Res. 113, C06011, doi:10.1029/2007JC004596.

Garrett, C., Kunze, E., 2007. Internal tide generation in the deep ocean. Annu. Rev. Fluid Mech. 39, 57-87.

Hurley, D.G., 1972. A general method for solving steady-state internal gravity wave problems. J. Fluid Mech. 56, 721-740.

Khatiwala, S., 2003. Generation of internal tides in an ocean of finite depth: analytical and numerical calculations. Deep-Sea Res. I 50, 3-21.

LeBlond, P.H., Mysak, L.A., 1978. Waves in the Ocean. Elsevier, Amsterdam.

Lighthill, J., 1967. On waves generated in dispersive systems by traveling forcing effects, with applications to the dynamics of rotating fluids. J. Fluid Mech. 27, 725-752.

Lighthill, J., 1978. Waves in Fluid. Cambridge University Press.

Munk, W.H., Wunsch, C.I., 1998. Abyssal recipes II: energetics of tidal and wind mixing. Deep-Sea Res. I 45, 1977-2010.

Nakamura, T., Awaji, T., Hatayama, T., Akitomo, K., Takizawa, T., Kono, T., Kawasaki, Y., Fukasawa, M., 2000. The generation of large-amplitude unsteady lee waves by subinertial K-1 tidal flow: a possible vertical mixing mechanism in the Kuril Straits. J. Phys. Oceanogr. 30, 1601-1621.

Nakamura, T., Awaji, T., 2001. The growth mechanism for topographic internal waves generated by an oscillatory flow. J. Phys. Oceanogr. 31, 2511-2524.

Nayfeh, A.H., 1973. Perturbation Methods. Wiley, New York.

Parsons, A.R., Bourke, R.H., Muench, R.D., Chiu, C.S., Lynch, J.F., Miller, J.H., Plueddemann, A.J., Pawlowicz, R., 1996. The Barents Sea Polar Front in summer. J. Geophys. Res. 101 (C6), 14201-14221.

Pisarev, S.V., 1996. Low-frequency internal waves near the shelf edge of the Arctic basin. Oceanology 36 (6), 771-779.

Smith, S.G.L., Young, W.R., 2002. Conversion of the barotropic tide. J. Phys. Oceanogr. 32, 1554-1566.

Smith, S.G.L., Young, W.R., 2003. Tidal conversion at a very steep ridge. J. Fluid Mech. 495, 175-191.

St. Laurent, L., Garrett, C., 2002. The role of internal tides in mixing the deep ocean. J. Phys. Oceanogr. 32, $2882-2899$.

Vallis, G.K., 2006. Atmospheric and Oceanic Fluid Dynamics: Fundamentals and Large-scale Circulation. Cambridge University Press.

Vlasenko, V., Stashchuk, N., Huntter, K., Sabinin, K., 2003. Nonlinear internal waves forced by tides near the critical latitude. Deep-Sea Res. I 50, 317-338. 
Voisin, B., 1991. Internal wave generation in uniformly stratified fluids. Part I. Green's function and point sources. J. Fluid Mech. 231, 439-480.

Van Haren, H., Millot, C., 2005. Gyroscopic waves in Mediterranean Sea. Geophys. Res. Lett. 32, L24614, doi:10.1029/2005GL023915. 